\title{
Impact of iodine concentration and scan parameters on image quality, contrast enhancement and radiation dose in thoracic CT
}

\author{
Marian S. Solbak', Mette K. Henning ${ }^{2}$, Andrew England ${ }^{3}$, Anne C. Martinsen ${ }^{1,4}$, Trond M. Aaløkken ${ }^{2,5}$ and
} Safora Johansen ${ }^{1,6^{*}}$ (i)

\begin{abstract}
Background: We investigated the impact of varying contrast medium (CM) densities and x-ray tube potentials on contrast enhancement (CE), image quality and radiation dose in thoracic computed tomography (CT) using two different scanning techniques.

Methods: Seven plastic tubes containing seven different CM densities ranging from of 0 to $600 \mathrm{HU}$ were positioned inside a commercial chest phantom with padding, representing three different patient sizes. Helical scans of the phantom in single-source mode were obtained with varying tube potentials from 70 to $140 \mathrm{kVp}$. A constant volume CT dose index (CTDlvol) depending on phantom size and automatic dose modulation was tested. CE (HU) and image quality (contrast-to-noise ratio, CNR) were measured for all combinations of CM density and tube potential. A reference threshold of CE and kVp was defined as $\geq 200 \mathrm{HU}$ and $120 \mathrm{kVp}$.

Results: For the medium-sized phantom, with a specific CE of 100-600 HU, the diagnostic CE (200 HU) at $70 \mathrm{kVp}$ was $\sim 90 \%$ higher than at $120 \mathrm{kVp}$, for both scan techniques $(p<0.001)$. Changes in CM density/specific HU together with lower $\mathrm{kVp}$ resulted in significantly higher CE and CNR $(p<0.001)$. When changing only the $\mathrm{kVp}$, no statistically significant differences were observed in CE or CNR $(p \geq 0.094)$, using both dose modulation and constant CTDIvol.
\end{abstract}

Conclusions: For thoracic CT, diagnostic CE $(\geq 200 \mathrm{HU})$ and maintained CNR were achieved by using lower CM density in combination with lower tube potential (< $120 \mathrm{kVp}$ ), independently of phantom size.

Keywords: Contrast media, Phantoms (imaging), Radiation dosage, Thorax, Tomography (x-ray computed)

\section{Background}

Computed tomography $(\mathrm{CT})$ enables detailed evaluation of the vascular system by obtaining contrast-enhanced scans. In thoracic CT, numerous vascular conditions such as aneurysms, haemorrhage, dissection and malformations require the administration of iodinated contrast medium

\footnotetext{
* Correspondence: Safora.johansen@oslomet.no

${ }^{1}$ Faculty of Health Sciences, Oslo Metropolitan University, Pilestredet 48, 0130 Oslo, Norway

${ }^{6}$ Department of Cancer Treatment, Oslo University Hospital, Oslo, Norway Full list of author information is available at the end of the article
}

(CM) to improve differentiation between normal anatomy and pathology [1-3]. Even though contrast-enhanced CT has become an important diagnostic tool, challenges still exist regarding exposure to ionising radiation and the use of iodinated CM. Exposure to ionising radiation is known to be carcinogenic and associated with an additional risk of cancer [4-6]. Thus, CT scanning must always be considered in accordance with the ALARA principle (as low as reasonably achievable) as regarding the radiation dose [4]. Patients with impaired renal function are especially at

\section{Springer Open}

() The Author(s). 2020 Open Access This article is licensed under a Creative Commons Attribution 4.0 International License, which permits use, sharing, adaptation, distribution and reproduction in any medium or format, as long as you give appropriate credit to the original author(s) and the source, provide a link to the Creative Commons licence, and indicate if changes were made. The images or other third party material in this article are included in the article's Creative Commons licence, unless indicated otherwise in a credit line to the material. If material is not included in the article's Creative Commons licence and your intended use is not permitted by statutory regulation or exceeds the permitted use, you will need to obtain permission directly from the copyright holder. To view a copy of this licence, visit http://creativecommons.org/licenses/by/4.0/. 
risk of developing post-contrast acute kidney injury caused by iodinated CM. Since the impact of both CM volume and renal $\mathrm{CM}$ concentration is still under debate, it is essential to reduce the risks through CM optimisation [7-9].

The degree of contrast enhancement (CE) is an important factor when determining the diagnostic image quality of a CT examination. This is particularly important when evaluating small structures such as the coronary arteries [10] or evaluating the presence of metastatic disease in lung parenchyma [11]. Three different factors have been shown to influence $\mathrm{CE}$ : acquisition parameters, patient physiology, and CM-related factors [12, 13]. Most important is the iodine delivery rate (i.e., the amount of iodine delivered per second) and the total iodine dose administered to the patient. For thoracic CT, high iodine concentrations ( $300 \mathrm{mg} \mathrm{I} / \mathrm{mL}$ and above) have routinely been administered to achieve diagnostic levels of $\mathrm{CE}$, but a higher iodine concentration itself does not result in a higher attenuation level when iodine delivery rate and total iodine dose are kept constant $[3,10,14]$.

Multi-detector CT scanners enable large volume coverages in a short time. Using high pitch in combination with wider detector ranges and shorter rotation time allows image acquisition at peak arterial CE before venous circulation impacts resultant image quality. This allows for more effective identification of hyper-vascular tumours [15]. However, fast scanning restricts the CM volume and consequently the $\mathrm{x}$-ray photon absorption. Alternatively, increasing iodine delivery rate may permit a reduction in $\mathrm{CM}$ volume and $\mathrm{CM}$ concentration, while maintaining diagnostic CE. A high iodine concentration is routinely used in CT angiography, to achieve opacification of around $250-300 \mathrm{HU}$ in the thoracic aorta, and $300-350 \mathrm{HU}$ in the coronary arteries $[3,10,12,13]$. The target opacification required for routine chest CT is typically lower (150-200 HU for thoracic vessels), but often depends on preferences of the supervising radiologist [3, $16,17]$.

Automatic tube voltage assistance techniques facilitate radiation dose reduction and improved visualisation of arteries. This is achieved by lowering the tube potential throughout the scan towards the k-shell energy level of iodine $(33.2 \mathrm{keV})$, thereby increasing photoelectric effect $[14,18-20]$. Scanning with a low tube potential and higher injection rate can allow a reduction of iodine concentration $[13,21]$ with the benefit of decreased contrast viscosity and a reduced risk of post-contrast acute kidney injury $[10,22]$. This method has been reported to reduce the radiation and iodine dose by between $40-$ $45 \%$ and $56-74 \%$, respectively, without loss of image quality [23-27].

Many studies have investigated the feasibility of "double-low" techniques which combine low tube voltage with low $\mathrm{CM}$ densities/volume and/or CM concentration for aortic, coronary and pulmonary $\mathrm{CT}$ angiography. These studies have repeatedly proved "doublelow" techniques to be beneficial by significantly reducing the iodine load and radiation dose [25, 28, 29]. However, to our knowledge, there is little known about the impact of CM densities/volume and varied tube potentials specifically for thoracic CT examinations. The aim of this study was to investigate the impact of variations in $\mathrm{CM}$ densities/volume for different tube potentials on contrast enhancement $(\mathrm{CE})$, overall image quality and radiation dose in thoracic $\mathrm{CT}$ examinations.

\section{Methods}

\section{Scan technique and phantom setup}

Seven drinking straws $(0.8 \mathrm{~cm}$ in diameter, $24 \mathrm{~cm}$ in length), containing mixtures of saline solution and iodinated CM, were used to simulate blood vessels. Straws were placed in a circular pattern peripherally in the lung of a commercial anthropomorphic chest phantom (N1 Lungman, Kyoto Kagaku Co., Tokyo, Japan) (Fig. 1a). The phantom has been described by Afadzi and colleagues [30] and Gomi et al. [31] and has been also reported in other studies [32,33]. Three phantom sizes were used. A set of anterior and posterior plates or "fat jackets" were added to simulate a large phantom $(26 \times$ $31 \mathrm{~cm}$ ), the anterior plate was removed to simulate a medium phantom $(23 \times 31 \mathrm{~cm})$, as illustrated in Fig. 1a, and no external plates were used for the small-sized phantom $(20 \times 27 \mathrm{~cm})$.

A series of CT scans were performed using a General Electric Revolution CT scanner (GE Healthcare, Waukesha, WI, USA). Phantoms were scanned at 70, 80, 100, 120 , and $140 \mathrm{kVp}$ at $0.5 \mathrm{~s} /$ rotation. Due to restrictions in tube output when employing a short acquisition-time and low tube potentials, the rotation time was increased to $1 \mathrm{~s} /$ rotation when scanning the medium phantom at $70 \mathrm{kVp}$ and scanning the large phantom at 70 and 80 $\mathrm{kVp}$. A total of $30 \mathrm{CT}$ acquisitions were performed with the scan parameters stated in Table 1 . The first 15 acquisitions were acquired with automatic dose modulation. The following 15 acquisitions were scanned with a fixed mean volume CT dose index (CTDIvol) of $7 \mathrm{mGy}$ (small phantom), $10 \mathrm{mGy}$ (medium phantom), and 17 mGy (large phantom), maintaining a near constant level of tube output per rotation. For the large phantom, only $13 \mathrm{mGy}$ was achieved for the $70 \mathrm{kVp}$ tube potential due to tube current limitations.

\section{Assessment of contrast enhancement}

Iodinated CM of $350 \mathrm{mg} \mathrm{I/mL} \mathrm{(Omnipaque,} \mathrm{Iohexol,}$ General Electric Healthcare, Oslo, Norway) was diluted in saline. When mixing the two components, equal amounts of saline were extracted, and CM was added to 


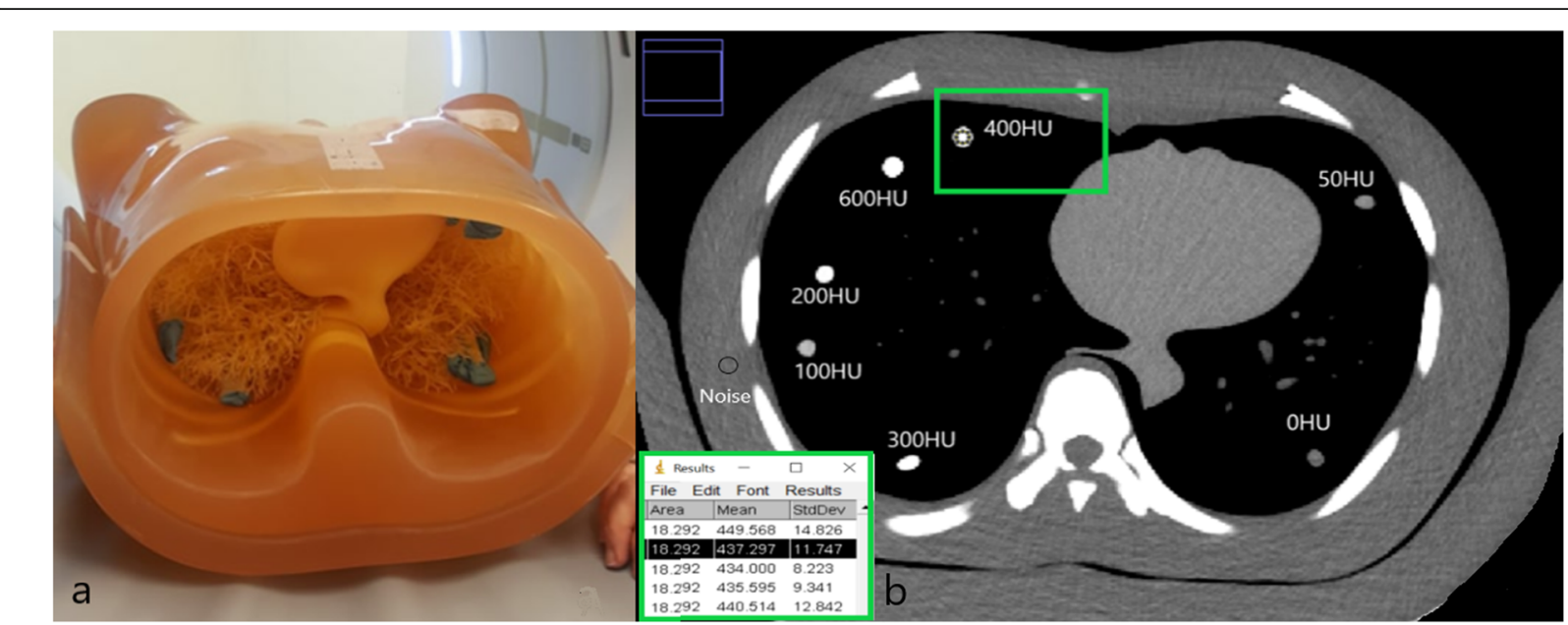

Fig. 1 a Kyoto Kaguka Lungman Phantom (medium size) displaying the seven straws. b A single computed tomography (CT) image acquired using the phantom. This image includes the seven straws containing different iodine densities, resulting in specific HU values at $120 \mathrm{kVp}$ when using a constant volume $\mathrm{CT}$ dose index

the prefilled $100 \mathrm{~mL}$ bottles of saline. At $120 \mathrm{kVp}, 1.2$ $\mathrm{mL}$ CM resulted in enhancement of $100 \mathrm{HU}$. Assuming a proportional relationship between $\mathrm{CM}$ and $\mathrm{HU}$, the amount of CM necessary to obtain different CE levels was calculated (Table 2). For simplicity, in the "Results" and "Discussion" sections, iodine concentrations/iodine density will only be referred to as HU values (a specific HU) as established in Table 2.

\section{Image quality assessment}

Image analyses were performed using the ImageJ software [34]. The ROIs were manually traced, and for each scan, a region of interest of $18.3 \mathrm{~mm}^{2}$ was placed in five slices (with $1 \mathrm{~cm}$ spacing) in each of the seven straws containing CM. The same approach was used to measure noise outside the lungs in the chest wall, in five slices (Fig. 1b). Measurements were repeated 35 times $(5 \times 7)$ for each scan and for the different tube potentials $(70-140 \mathrm{kVp})$ and phantom sizes making a total of 600 circular regions of interest (Fig. 1). Image quality was assessed by calculating contrast-to-noise ratio (CNR) [35]. This study refers to enhancement of $200 \mathrm{HU}$ and $120 \mathrm{kVp}$ [3] as reference level. Images with $\mathrm{CE} \geq 200 \mathrm{HU}$ were considered diagnostically acceptable for thoracic CT scans $[3,13,17,36,37]$.

\section{Dose assessment}

The CTDIvol reported on the scanner after exposure was noted for each scan. The hospital CT quality assurance programme was carried out twice in 2018 and once in December 2019, by CT physicists. Measured CTDI in air was on all the three occasions within $10 \%$ from the

Table 1 Details of the contrast medium specifics

\begin{tabular}{|c|c|c|c|c|c|c|c|c|c|c|c|}
\hline \multirow{2}{*}{$\begin{array}{l}\text { Phantom } \\
\text { size }\end{array}$} & \multirow{2}{*}{$\begin{array}{l}\text { Scan parameter } \\
\text { tube voltage: }\end{array}$} & \multicolumn{5}{|c|}{ Constant CTDI } & \multicolumn{5}{|c|}{ Dose modulation } \\
\hline & & $70 \mathrm{kVp}$ & $80 \mathrm{kVp}$ & $100 \mathrm{kVp}$ & $120 \mathrm{kVp}$ & $140 \mathrm{kVp}$ & $70 \mathrm{kVp}$ & $80 \mathrm{kVp}$ & $100 \mathrm{kV}$ & $120 \mathrm{kV}$ & $140 \mathrm{kV}$ \\
\hline \multirow[t]{3}{*}{ Large } & Rotation time (s) & 1 & 1 & 0.5 & 0.5 & 0.5 & 0.5 & 0.5 & 0.5 & 0.5 & 0.5 \\
\hline & mAs (range 80-500) & 500 & 395 & 405 & 250 & 170 & 499 & 499 & 464 & 372 & 282 \\
\hline & CTDIvol (mGy) & 13.17 & 16.6 & 16.68 & 16.6 & 16.43 & 6.59 & 10.11 & 14.79 & 16.6 & 17.59 \\
\hline \multirow[t]{3}{*}{ Medium } & Rotation time (s) & 1 & 0.5 & 0.5 & 0.5 & 0.5 & 0.5 & 0.5 & 0.5 & 0.5 & 0.5 \\
\hline & mAs (range 80-500) & 380 & 475 & 240 & 150 & 105 & 499 & 499 & 322 & 244 & 178 \\
\hline & CTDlvol (mGy) & 10 & 9.98 & 9.89 & 9.96 & 10.15 & 6.09 & 8.14 & 8.98 & 10 & 11.6 \\
\hline \multirow[t]{3}{*}{ Small } & Rotation time (s) & 0.5 & 0.5 & 0.5 & 0.5 & 0.5 & 0.5 & 0.5 & 0.5 & 0.5 & 0.5 \\
\hline & mAs (range 80-500) & 500 & 330 & 170 & 105 & 70 & 484 & 630 & 219 & 106 & 123 \\
\hline & CTDIvol (mGy) & 6.59 & 6.93 & 7.00 & 6.97 & 6.77 & 4.67 & 5.18 & 5.73 & 7.05 & 8.75 \\
\hline
\end{tabular}

CTDIvol Volume computed tomography dose index. Scan technique: constant CTDlvol and dose modulation (SmartmA) using conventional, iterative reconstruction. Additional parameters: scan mode, helical; detector collimation, $80 \times 0.625$; pitch, 0.5 ; scan field of view, $50 \mathrm{~cm}$; display field of view, $36 \mathrm{~cm}$; reconstructed slice thickness, $2.5 \mathrm{~mm}$; reconstruction kernel, standard; iterative reconstruction, adaptive statistical iterative reconstruction, ASIR-V 50\%; noise index14.5 (scans with dose modulation) 
Table 2 Details of the computed tomography scanning parameters

\begin{tabular}{lll}
\hline $\begin{array}{l}\text { lodine densities } \\
(\mathrm{mg} \mathrm{I} / \mathrm{mL} \rightarrow \mathrm{HU})\end{array}$ & $\begin{array}{l}\mathrm{CM} \text { volume } \\
(350 \mathrm{mg} \mathrm{L} / \mathrm{mL})\end{array}$ & $\begin{array}{l}\text { Mixing ratio } \\
(\mathrm{CM}: \text { Saline solution })\end{array}$ \\
\hline $0 \rightarrow 0$ & $0 \mathrm{~mL}$ & $0: 1$ \\
$2.1 \rightarrow 50$ & $0.6 \mathrm{~mL}$ & $1: 165$ \\
$4.2 \rightarrow 100$ & $1.2 \mathrm{~mL}$ & $1: 82$ \\
$8.4 \rightarrow 200$ & $2.4 \mathrm{~mL}$ & $1: 41$ \\
$12.6 \rightarrow 300$ & $3.6 \mathrm{~mL}$ & $1: 27$ \\
$18.2 \rightarrow 400$ & $5.2 \mathrm{~mL}$ & $1: 18$ \\
$26.6 \rightarrow 600$ & $7.6 \mathrm{~mL}$ & $1: 12$ \\
\hline
\end{tabular}

CM Contrast medium

vendor's technical specification, and according to the institutions' protocol for quality assurance, CTDIvol is then assumed to be within similar acceptance.

\section{Statistical analysis}

Analysis was performed using SPSS Version 26 (IBM Inc, Armonk, NY, USA). Data was presented as mean \pm standard deviation, minimum and maximum values. The non-parametric Kruskal-Wallis analysis of variance by ranks was also used to compare the difference in objective image quality. The $p$ values $<0.05$ were considered as statistically significant.

\section{Results}

\section{CE assessment}

The mean diagnostic CE was higher for all CM densities and all three phantom sizes at lower tube potentials $(70-100 \mathrm{kVp})$ when compared to the reference tube voltage of $120 \mathrm{kVp}$ (Fig. 2). The diagnostic CE at $70 \mathrm{kVp}$ and $80 \mathrm{kVp}$ was $91 \%$ and $59 \%$ higher, respectively, when compared to acquisitions at $120 \mathrm{kVp}$. This was seen for both acquisitions using dose modulation, and for those with a constant CTDIvol for the reference CM density at $200 \mathrm{HU}$ (Fig. 2). Our results show that using lower CM densities/specific $\mathrm{HU}$ at lower tube potentials (e.g., 70 $\mathrm{kVp}$ ) will result in higher diagnostic CE compared to acquisitions at $120 \mathrm{kVp}$ (Fig. 2). These differences were statistically significant $(p<0.001)$. There was no significant difference in the measured $\mathrm{CE}$ between phantom sizes $(p \geq 0.494)$.

\section{Image quality assessment}

For the medium-sized phantom, at the reference level $(120 \mathrm{kVp}, 200 \mathrm{HU}), \mathrm{CNR}$ started at 30 and 23 for the images obtained at constant CTDIvol and dose modulation, respectively, (Fig. 3). At the lowest tube voltage of 70 $\mathrm{kVp}$, our results showed an increase in the CNR of $80 \%$ for the 200-HU CE using dose modulation, compared to the reference at $120 \mathrm{kVp}$. When using constant CTDIvol, our results showed that CNR was higher (96\%) for 200
HU CE at $70 \mathrm{kVp}$ versus $120 \mathrm{kVp}$. The higher CNR at lower tube voltages was seen for all measurements carried out in this study, independently of the scan technique and phantom size (Fig. 3).

Changes following lower $\mathrm{CM}$ density/specific $\mathrm{HU}$, resulted in significantly higher CE and CNR $(p<0.001)$. However, when changing the tube potential, no significant differences (for the same CM density/specific $\mathrm{HU}$ ) were observed in CE or CNR $(p>0.094)$. Again, this was seen for both dose modulation and for a constant CTDIvol (Figs. 2 and 3).

\section{Radiation dose}

For scans with constant CTDIvol, the radiation dose was 7, 10, and $17 \mathrm{mGy}$ for small, medium and large phantoms across the various tube potentials (Table 1). However, for the large phantom, the CTDIvol was 13 instead of 17 when scanning with $70 \mathrm{kVp}$ due to technical limitations of the CT scanner. The radiation dose, when dose modulation was applied for the medium-sized phantom, showed a reduction of $10 \%, 19 \%$, and $39 \%$ for $100 \mathrm{kVp}, 80 \mathrm{kVp}$, and $70 \mathrm{kVp}$, respectively when compared to the reference tube voltage $(120 \mathrm{kVp})$ (Table 1$)$. For the large phantom, the radiation dose decreased with $11 \%, 39 \%$, and $60 \%$ at $100 \mathrm{kVp}, 80 \mathrm{kVp}$, and $70 \mathrm{kVp}$. Correspondingly, a dose reduction of $19 \%, 27 \%$, and $34 \%$ was observed for the small phantom, across the respective tube potentials. Note that due to tube output limitations, the mAs was cut at a certain level for the large phantom at 70 and $80 \mathrm{kVp}$ and the medium phantom at $70 \mathrm{kVp}$, resulting in lower doses than expected.

\section{Discussion}

In this study, the impact of varying CM densities at different tube potentials on the overall image quality and diagnostic CE for thoracic CT was investigated. Our results show that increasing $\mathrm{CM}$ densities leads to significantly higher diagnostic $\mathrm{CE}$ and image quality (CNR). While a reduction in tube potential was shown to increase CE and CNR, these differences were not statistically significant, regardless of employed scan technique or patient size.

The clear benefits of low- $\mathrm{kV}$ protocols have been a favoured topic, with resulting radiation and $\mathrm{CM}$ dose savings between 40 and $60 \%$ reported, when reducing tube potential from 120 to $80 \mathrm{kVp}$ [23-27]. Further dose savings and improved image quality have been reported, when combining low $\mathrm{kV}$ and optimised injection protocols which would reduce $\mathrm{CM}$ volume or concentration $[14,28,36,38]$. The latter is demonstrated in the present phantom-based study. Reducing the tube voltage from 120 to $70 \mathrm{kVp}$ for reference diagnostic CE of $200 \mathrm{HU}$ resulted in increases in CNR of up to $80 \%$ and $96 \%$ using dose modulation and constant CTDIvol, respectively, in 


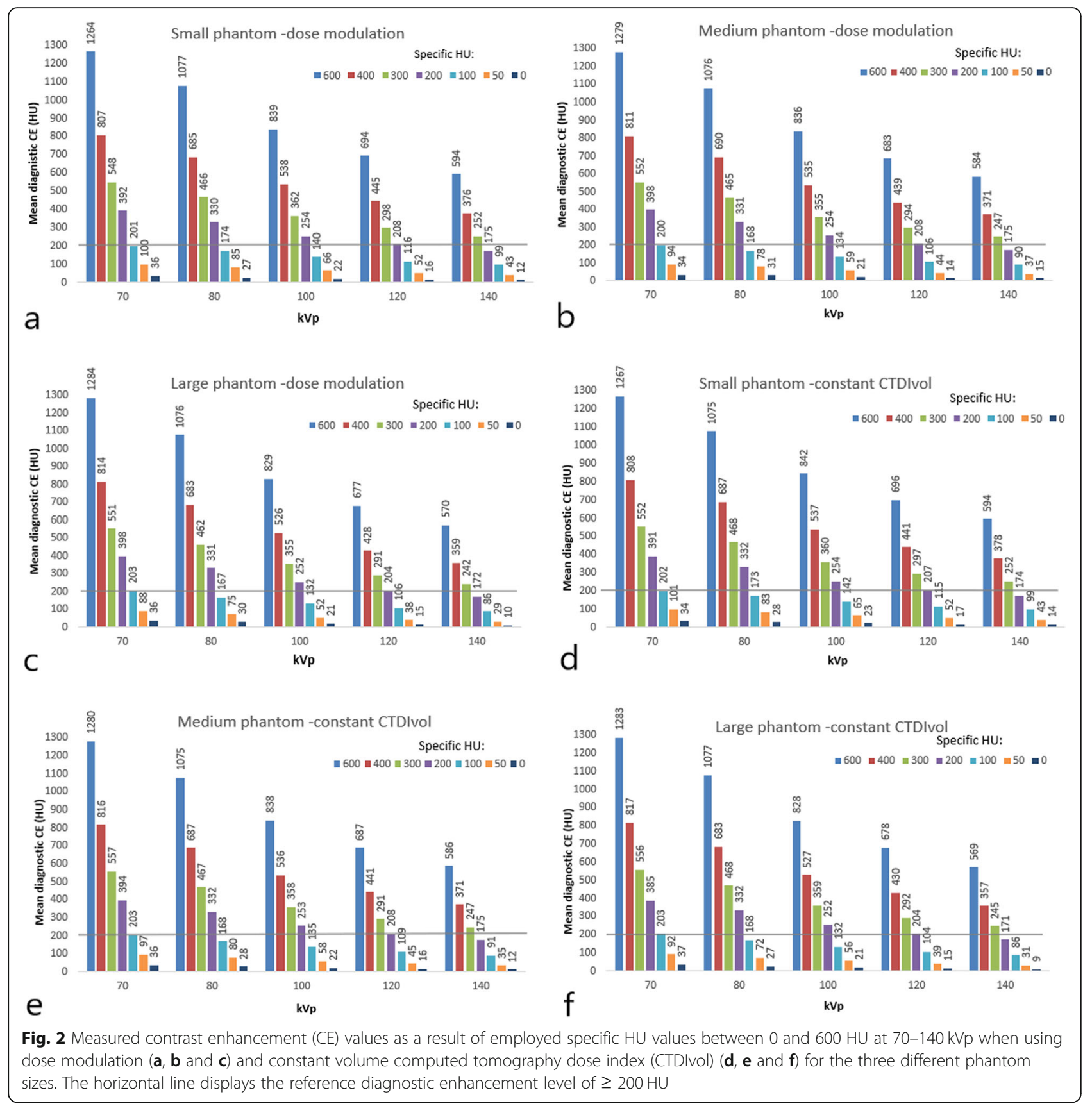

the medium phantom size. When lowering the tube potential, there is a debate regarding which approach is optimal for lowering the CM dose. Fleischmann et al. [14] have reported moderate iodine concentrations $(300 \mathrm{mg}$ $\mathrm{I} / \mathrm{mL}$ ) to be superior to higher CM concentrations (400 $\mathrm{mg} \mathrm{I} / \mathrm{mL}$ ) for a constant iodine delivery rate when using $70 \mathrm{kVp}$ to achieve sufficient CE over $300 \mathrm{HU}$ [14]. The "double-low" approach has been favoured by several earlier studies [14, 28, 38-40] because of resultant lower effective doses and iodine doses with comparable image quality. According to the literature, a $\mathrm{CE} \geq 200 \mathrm{HU}$ in the thoracic region is clinically acceptable [3, 13]. For a routine chest CT, $60-70 \mathrm{~mL}$ of $350-370 \mathrm{mg} \mathrm{I} / \mathrm{mL} \mathrm{CM}$ has been suggested to be acceptable to achieve a $\mathrm{CE}$ of $150-200 \mathrm{HU}$ [3].

The results of our study, despite investigating the impact of CM density on enhancement instead of CM concentration used in other studies, confirm the same tendency as reported in the study by Sun et al. [28]. They suggested greater potential for lowering the iodine load even in obese patients by $27 \%$, scanning with 100 $\mathrm{kVp}$ instead of $120 \mathrm{kVp}$. Our investigation has reported a $50 \%$ reduction of $\mathrm{CM}$ density (medium phantom size) for the same diagnostic CE $(200 \mathrm{HU})$ and CNR, by 


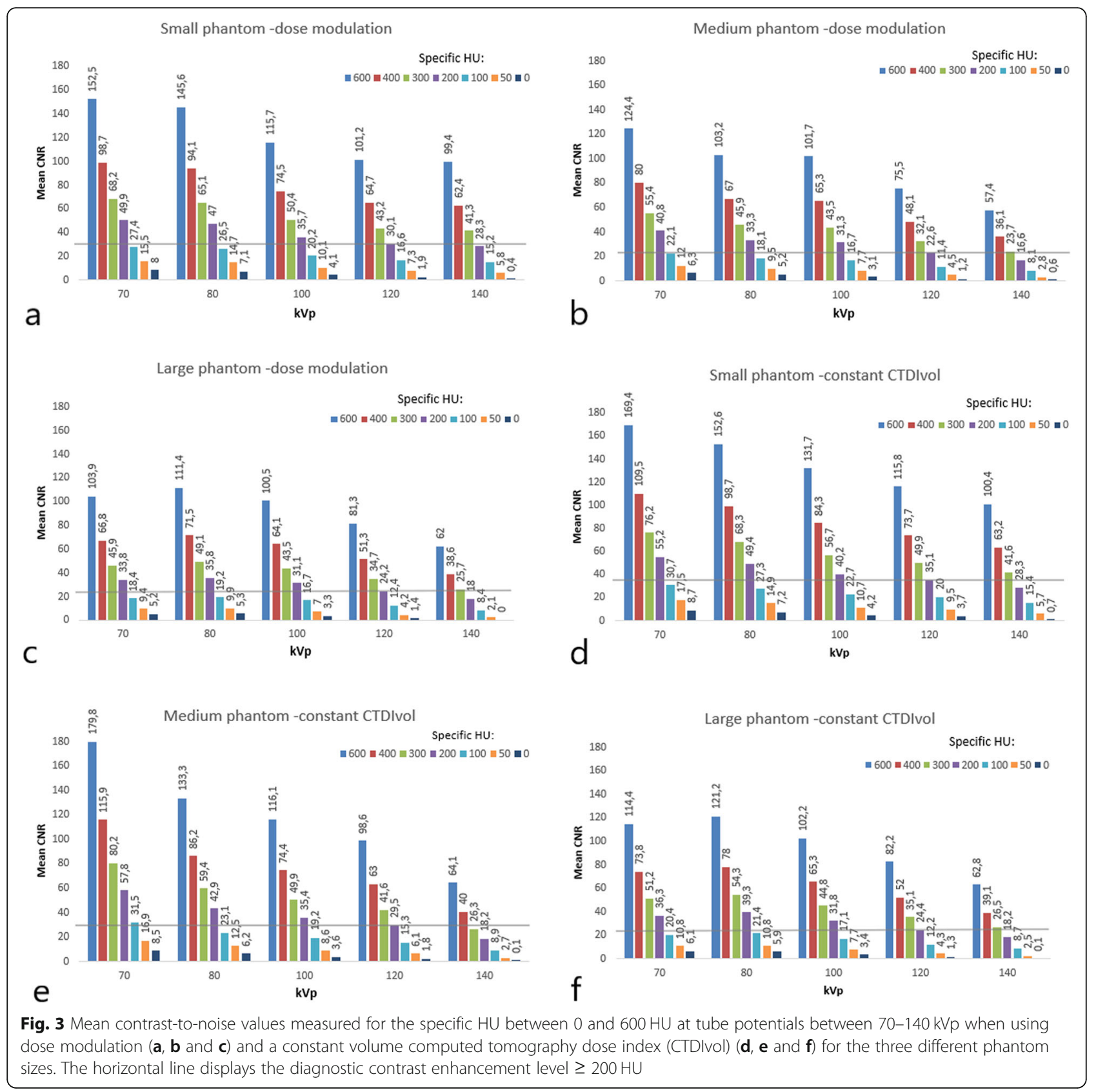

reducing tube potential from 120 to $70 \mathrm{kVp}$, using dose modulation as shown in Figs. 2 and 3. Our findings are also in agreement with the $51 \% \mathrm{CM}$ density reduction reported by Thor et al. [27]. However, larger patient sizes as demonstrated in our study (see Fig. 3) may be a limitation with regards to the required tube output needed to achieve comparable CNR levels when using higher $\mathrm{kVp}$ values. Van Hamersvelt et al. [26] have shown a similar $40-60 \%$ iodine reduction, without loss of image quality using dual source and dual energy CT. In our study, a single source and single energy CT protocol was employed.
The CNR is primarily affected by CM signal and image noise, broadly becoming the most appropriate measure for investigating iodine-enhanced vessels and structures $[27,40]$. When increasing contrast enhancement, by lowering photon energy towards the k-shell electron binding energy of iodine, more noise is accepted [41]. As shown in Fig. 3, the CNR increased from 22.6 to 40.8 , approximately $80 \%$, for the medium-sized phantom, when tube potential was reduced from 120 to $70 \mathrm{kVp}$ (see Fig. 3). CNR (22.6 to 40.8 ) increased by $55.4 \%$ when using a fixed CTDIvol, inherently improving the image quality (see Fig. 3). However, these differences in CNR 
were not statistically significant, when using both scan techniques $(p<0.094)$. Patient size nevertheless has a great impact on image noise as photon penetration decreases in larger patients and a higher $\mathrm{x}$-ray beam energy is required to achieve the same noise level [36, 39]. In our study, the image quality remained diagnostically acceptable independent of phantom size.

The CNR values resulting in a diagnostic CE $\geq 200$ HU were above 23 and 30, with and without dose modulation, for the medium-sized phantom (Fig. 3). When compared to constant CTDIvol, dose modulation continuously reduces the tube current to patient/phantom attenuation profile, while maintaining a given noise index. This may cause an effective dose reduction of $53 \%$ according to Kok et al. [24] which supports our observation with only a slight change in CNR using dose modulation compared to constant CTDIvol (Fig. 3).

There are several limitations in our study. This was a phantom study, thus, no anatomical noise or artefacts caused by breathing and pulsation were present in the images. Furthermore, for the fixed parameter settings, the CTDIvol was lower for the 70 and 80 $\mathrm{kVp}$ levels for the large phantom due to technical limitations. Still, the systematic evaluation of different $\mathrm{CM}$ concentrations in the different phantom sizes for different scan techniques and dose levels would not be possible to obtain in a clinical setting due to patient radiation dose issues. Thus, performing a phantom study as the first step of systematic evaluation prior to a clinical study is needed to fully assess different scan techniques and available parameter settings. Each ROI placed inside the plastic straws and the chest wall was separated by air, influencing the calculation of objective image quality. No assessment of subjective image quality was conducted in this study. Therefore, to fully assess and validate the findings in this study, clinical studies including both objective and subjective image quality evaluation are needed to confirm our findings in routine clinical care. However, our results show that increased $\mathrm{CE}$ at lower tube voltages can be employed in clinical practice.

In conclusion, this study demonstrated that the combination of lower CM densities (specific HU) combined with lower tube potentials $(e . g$., $70 \mathrm{kVp})$ resulted in improved CE enhancement ( 90\% higher), and maintained image quality (80\% higher CNR) in chest $\mathrm{CT}$ when compared to acquisitions at $120 \mathrm{kVp}$. Using double-low method in thoracic CT examinations, CM density can be reduced by approximately $50 \%$ while maintaining CNR. Our findings were independent of scan technique and phantom size. To fully assess the potential of reduced CM densities for lower $\mathrm{kVp}$ in chest $\mathrm{CT}$, clinical validation of the results from this study are needed.

\section{Abbreviations}

CE: Contrast enhancement; CM: Contrast medium; CNR: Contrast-to-noise ratio; CT: Computed tomography; CTDIvol: Volume CT dose index

\section{Authors' contributions}

MSS carried out data collection and drafted the manuscript. MKH contributed to data collection and data quality assurance. AE, TMA, and ACM participated in the drafting of the manuscript and review. SJ led the research team, contributed to data collection, data analysis, carried out study design, contributed to manuscript finalisation, and contributed to the revision. All authors read and approved the final manuscript.

\section{Funding information}

None

\section{Availability of data and materials}

The datasets used and/or analysed during the current study are available from the corresponding author on reasonable request

Ethics approval and consent to participate

Not applicable

Consent for publication

Not applicable

\section{Competing interests}

None

\section{Author details}

${ }^{1}$ Faculty of Health Sciences, Oslo Metropolitan University, Pilestredet 48, 0130 Oslo, Norway. ${ }^{2}$ Department of Radiology and Nuclear Medicine, Oslo University Hospital, Oslo, Norway. ${ }^{3}$ School of Allied Health Professions, Keele University, Staffordshire, England. ${ }^{4}$ Department of Diagnostic Physics, Oslo University Hospital, Oslo, Norway. ${ }^{5}$ Faculty of Medicine, University of Oslo, Oslo, Norway. 'Department of Cancer Treatment, Oslo University Hospital, Oslo, Norway.

Received: 6 April 2020 Accepted: 12 August 2020

Published online: 11 September 2020

\section{References}

1. McMahon MA, Squirrell CA (2010) Multidetector CT of aortic dissection: a pictorial review. Radiographics 30:445-460. https://doi.org/10.1148/rg.302095104

2. Whiting P, Singatullina N, Rosser J (2015) Computed tomography of the chest: I. basic principles. BJA Education 15:299-304. https://doi.org/10.1093/ bjaceaccp/mku063

3. Bae KT (2010) Optimization of contrast enhancement in thoracic MDCT. Radiol Clin North Am 48:9-29. https://doi.org/10.1016/j.rcl.2009.08.012

4. Dahal S, Budoff MJ (2019) Low-dose ionizing radiation and cancer risk: not so easy to tell. Quant Imaging Med Surg 9:2023-2026. https://doi.org/10. 21037/qims.2019.10.18

5. Duncan JR, Lieber MR, Adachi N, Wahl RL (2018) Radiation dose does matter: mechanistic insights into DNA damage and repair support the linear nothreshold model of low-dose radiation health risks. J Nucl Med 59:1014-1016. https://doi.org/10.2967/jnumed.118.210252

6. Mavragani IV, Nikitaki Z, Kalospyros SA, Georgakilas AG (2019) lonizing radiation and complex DNA damage: from prediction to detection challenges and biological significance. Cancers (Basel) 11:1789. https://doi. org/10.3390/cancers 11111789

7. McCullough PA, Choi JP, Feghali GA et al (2016) Contrast-induced acute kidney injury. J Am Coll Cardiol 68:1465-1473. https://doi.org/10.1016/j.jacc.2016.05.099

8. van der Molen AJ, Reimer P, Dekkers IA et al (2018) Post-contrast acute kidney injury-part 1: definition, clinical features, incidence, role of contrast medium and risk factors. Eur Radiol 28:2845-2855. https://doi.org/10.1007/ s00330-017-5246-5

9. Yessayan L, Heung M (2018) Contrast-induced acute kidney injury-nephrology perspective. Clin Nephrol 89:170-175. https://doi.org/10.5414/CN109259

10. Mihl C, Wildberger JE, Jurencak T et al (2013) Intravascular enhancement with identical iodine delivery rate using different iodine contrast media in a circulation phantom. Invest Radiol 48:813-818. https://doi.org/10.1097/RLI. Ob013e31829979e8 
11. Raj V, Kirke R, Bankart MJ, Entwisle JJ (2011). Multidetector CT imaging of pleura: comparison of two contrast infusion protocols. Br J Radiol 84:796799. https://doi.org/10.1259/bjr/55980445

12. Bae KT, Heiken JP (2005) Scan and contrast administration principles of MDCT. Eur Radiol Suppl 15:46-59. https://doi.org/10.1007/s10406-005-0165-y

13. Weininger M, Barraza JM, Kemper CA, Kalafut JF, Costello P, Schoepf UJ (2011) Cardiothoracic CT angiography: current contrast medium delivery strategies. AJR Am J Roentgenol 196:260-272. https:/doi.org/10.2214/AJR.10.5814

14. Fleischmann U, Pietsch $H$, Korporaal JG et al (2018) Impact of contrast media concentration on low-kilovolt computed tomography angiography: a systematic preclinical approach. Invest Radiol 53:264-270. https://doi.org/10. 1097/RLI.0000000000000437

15. Faggioni L, Gabelloni M (2016) lodine concentration and optimization in computed tomography angiography: current issues. Invest Radiol 51:816822. https://doi.org/10.1097/RLI.0000000000000283

16. Ibrahim N, Karim NA, Shuaib I, Osman N, Hashim S, Phuah H (2019) Comparison study between different contrast administration protocols for routine CT thorax examination in two tertiary centres. J Phys Conf Ser 1248 : 1-6. https://doi.org/10.1088/1742-6596/1248/1/012028

17. Colip CG, Gorantla V, LeBedis CA, Soto JA, Anderson SW (2017) Extremity CTA for penetrating trauma: 10-year experience using a 64detector row CT scanner. Emerg Radiol 24:223-232. https://doi.org/10. 1007/s10140-016-1469-z

18. Bogot NR, Fingerle A, Shaham D, Nissenbaum I, Sosna J (2011) Image quality of low-energy pulmonary CT angiography: comparison with standard CT. AJR Am J Roentgenol 197:273-278. https://doi.org/10.2214/AJR.10.5318

19. Meyer M, Haubenreisser $H$, Schoepf UJ et al (2014) Closing in on the K edge: coronary CT angiography at 100, 80, and $70 \mathrm{kV}$-initial comparison of a second-versus a third-generation dual-source CT system. Radiology 273: 373-382. https://doi.org/10.1148/radiol.14140244

20. Annoni AD, Mancini ME, Andreini D et al (2017) Overall evaluability of low dose protocol for computed tomography angiography of thoracic aorta using $80 \mathrm{kV}$ and iterative reconstruction algorithm using different concentration contrast media. J Med Imaging Radiat Oncol 61:614-621. https://doi.org/10.1111/1754-9485.12608

21. Tan SK, Ng KH, Yeong CH et al (2019) Personalized administration of contrast medium with high delivery rate in low tube voltage coronary computed tomography angiography. Quant Imaging Med Surg 9:552-564. https://doi.org/10.21037/qims.2019.03.13

22. Buls N, Van Gompel G, Van Cauteren T et al (2015) Contrast agent and radiation dose reduction in abdominal $\mathrm{CT}$ by a combination of low tube voltage and advanced image reconstruction algorithms. Eur Radiol 25:10231031. https://doi.org/10.1007/s00330-014-3510-5

23. lyama Y, Nakaura T, Yokoyama K et al (2016) Low-contrast and low-radiation dose protocol in cardiac computed tomography: usefulness of low tube voltage and knowledge-based iterative model reconstruction algorithm. J Comput Assist Tomogr 40:941-947. https://doi.org/10.1097/RCT.0000000000000440

24. Kok M, Mihl C, Hendriks BM et al (2016) Optimizing contrast media application in coronary $C T$ angiography at lower tube voltage: evaluation in a circulation phantom and sixty patients. Eur J Radiol 85:1068-1074. https:// doi.org/10.1016/j.ejrad.2016.03.022

25. Wei L, Li S, Gao Q, Liu Y, Ma X (2016) Use of low tube voltage and low contrast agent concentration yields good image quality for aortic $C T$ angiography. Clin Radiol. 71:5-10. https://doi.org/10.1016/j.crad.2016.07.018

26. Van Hamersvelt RW, Eijsvoogel NG, Mihl C et al (2018) Contrast agent concentration optimization in CTA using low tube voltage and dual-energy CT in multiple vendors: a phantom study. Int J Cardiovasc Imaging 34:12651275. https://doi.org/10.1007/s10554-018-1329-x

27. Thor D, Brismar TB, Fischer MA (2015) Low tube voltage dual source computed tomography to reduce contrast media doses in adult abdomen examinations: a phantom study. Med Phys 42:5100-5109. https://doi.org/10. $1118 / 1.4927791$

28. Sun G, Hou Y, Zhang B et al (2015) Application of low tube voltage coronary CT angiography with low-dose iodine contrast agent in patients with a BMI of 26-30 kg/m2. Clin Radiol 70:138-145. https://doi.org/10.1016/j. crad.2014.10.002

29. Talei Franzesi CR, Ippolito D, Riva L, Fior D, Cangiotti C, Sironi S (2018) Diagnostic value of iterative reconstruction algorithm in low kV CT angiography (CTA) with low contrast medium volume for transcatheter aortic valve implantation (TAVI) planning: image quality and radiation dose exposure. Br J Radiol 91:1092. https://doi.org/10.1259/bjr.20170802
30. Afadzi M, Fosså K, Andersen HK, Aaløkken TM, Martinsen ACT (2020) Image quality measured from ultra-low dose chest computed tomography examination protocols using 6 different iterative reconstructions from 4 vendors, a phantom study. J Comput Assist Tomogr. 44:95-101. https://doi. org/10.1097/RCT.0000000000000947

31. Gomi T, Nakajima M, Fujiwara H et al (2012) Comparison between chest digital tomosynthesis and CT as a screening method to detect artificial pulmonary nodules: a phantom study. Br J Radiol 85:622-629. https://doi. org/10.1259/bjr/12643098

32. Doo K, Kang E-Y, Yong H-S, Woo OH, Lee KY, Oh YW (2014) Accuracy of lung nodule volumetry in low-dose $C T$ with iterative reconstruction: an anthropomorphic thoracic phantom study. Br J Radiol 87:1041. https://doi. org/10.1259/bjr.20130644

33. Jin L, Sun Y, Li M (2019) Use of an anthropomorphic chest model to evaluate multiple scanning protocols for high-definition and standarddefinition computed tomography to detect small pulmonary nodules. Med Sci Mon 25:2195. https://doi.org/10.12659/MSM.913243

34. Schneider CA, Rasband WS, Eliceiri KW (2012) NIH image to imageJ: 25 years of image analysis. Nat Methods 9:671-675. https://doi.org/10.1038/nmeth.2089

35. Hendriks BM, Eijsvoogel NG, Kok M (2018) Optimizing pulmonary embolism computed tomography in the age of individualized medicine: a prospective clinical study. Invest Radiol 53:306-312. https://doi.org/10.1097/RLI. 0000000000000443

36. Mühlenbruch G, Behrendt FF, Eddahabi MA et al (2018) Which iodine concentration in chest CT?-a prospective study in 300 patients. Eur Radiol 18:2826. https://doi.org/10.1007/s00330-008-1080-0

37. Caruso D, Eid M, Schoepf UJ et al (2017) Optimizing contrast media injection protocols in computed tomography angiography at different tube voltages: evaluation in a circulation phantom. J Comput Assist Tomogr 41: 804-810. https://doi.org/10.1097/RCT.0000000000000613

38. Szucs-Farkas Z, Verdun FR, von Allmen G, Mini RL, Vock P (2008) Effect of Xray tube parameters, iodine concentration, and patient size on image quality in pulmonary computed tomography angiography: a chestphantom-study. Invest Radiol 43:374-381. https://doi.org/10.1097/RLI. Ob013e3181690042

39. Aschoff AJ, Catalano C, Kirchin MA, Krix M, Albrecht T (2017) Low radiation dose in computed tomography: the role of iodine. Br J Radiol 90:20170079. https://doi.org/10.1259/bjr.20170079

40. Kok M, Mihl C, Seehofnerová A et al (2015) Automated tube voltage selection for radiation dose reduction in CT angiography using different contrast media concentrations and a constant iodine delivery rate. AJR Am J Roentgenol 205:1332-1138. https://doi.org/10.2214/AJR.14.13957

41. García-Garrigós E, Arenas-Jiménez JJ, Sánchez-Payá J (2018) Best protocol for combined contrast-enhanced thoracic and abdominal CT for lung cancer: a single-institution randomised crossover clinical trial. AJR Am J Roentgenol 2018:1226-1234. https://doi.org/10.2214/AJR.17.19185

\section{Publisher's Note}

Springer Nature remains neutral with regard to jurisdictional claims in published maps and institutional affiliations.

\section{Submit your manuscript to a SpringerOpen ${ }^{\circ}$ journal and benefit from:}

- Convenient online submission

- Rigorous peer review

- Open access: articles freely available online

- High visibility within the field

- Retaining the copyright to your article

Submit your next manuscript at $>$ springeropen.com 